\title{
Setaria marsballi Boulenger 1921, et Setaria marshalli pandei n. s.-sp.
}

par C. SHOHO (1)

L'identification des Setaria est devenue importante et intéressante pour l'étude des Nématodes depuis le travail japonais sur « la nématodiase cérébrospinale » ou paralysie lombaire des moutons, chèvres et chevaux (voir Yamagiwa et Shoho 1944 ; Innes et Shoho 1953 ; Shoho 1959, ainsi que plusieurs publications originaires d'autres pays : Bush 1951 ; Sprent 1955 ; Cadenacii 1956 et Yeh 1959). Les nombreuses publications récentes traitant du parasitisme accidentel par divers Nématodes d'hôtes anormaux indiquent l'intérêt qu'il faut attacher à l'identification de ces Helminthes.

Pour tenter de trancher entre les diverses opinions émises sur l'identité de quelquesuns de ces Setaria, nous avons commencé à étudier, depuis 1952, les Setaria des Herbivores, en particulier des animaux domestiques. Quelques travaux ont été publiés antérieurement sur les Setaria d'Herbivores sauvages, tels que Axis A. ceylonensis (Shoho 1958, a), Alces A. gigas en Amérique du Nord (Shoho 1958, b) et Capreolus C. en Suisse (1959). Ces espèces faisaient l'objet de discussions quant à leur identité avec les Setaria des animaux domestiques (Baylis 1936).

Le présent travail concerne une espèce du genre Setaria, mal connue dans la littérature occidentale, et sa sous-espèce qui est nouvelle.

Pendant un court séjour en Inde septentrionale, au printemps de 1963, le Professeur G.-P. Pande, à Mathura, nous a confié deux lots de Setaria, l'un provenant d'un veau bubalin âgé d'un mois, l'autre de veaux bubalins âgés de 4 à 6 mois. En examinant ces spécimens au Japon, nous avons observé qu'ils étaient presque identiques à Setaria marshalli, à l'exception de différences, faibles, mais constantes, dans la forme des appendices latéraux (= phasmides) de l'extrémité postérieure des femelles, et de variations dans la taille de ces femelles. Cette espèce était considérée par quelques travailleurs comme non valide parce que décrite, pensaient-ils, à partir d'exemplaires abîmés d'une espèce ordinairement trouvée chez les bœufs domestiques; en réalité, nous l'avons reconnue au Japon comme une espèce indépendante, vivant habituellement chez les

(1) Département de Pathologie. Faculté Vétérinaire, Université de Khartoum (Soudan). Depuis décembre 1963, Department of Parasitology, University of Hawaii (Honolulu). Actuellement: Nakayama-Soen - Takarazuka - Japon. 

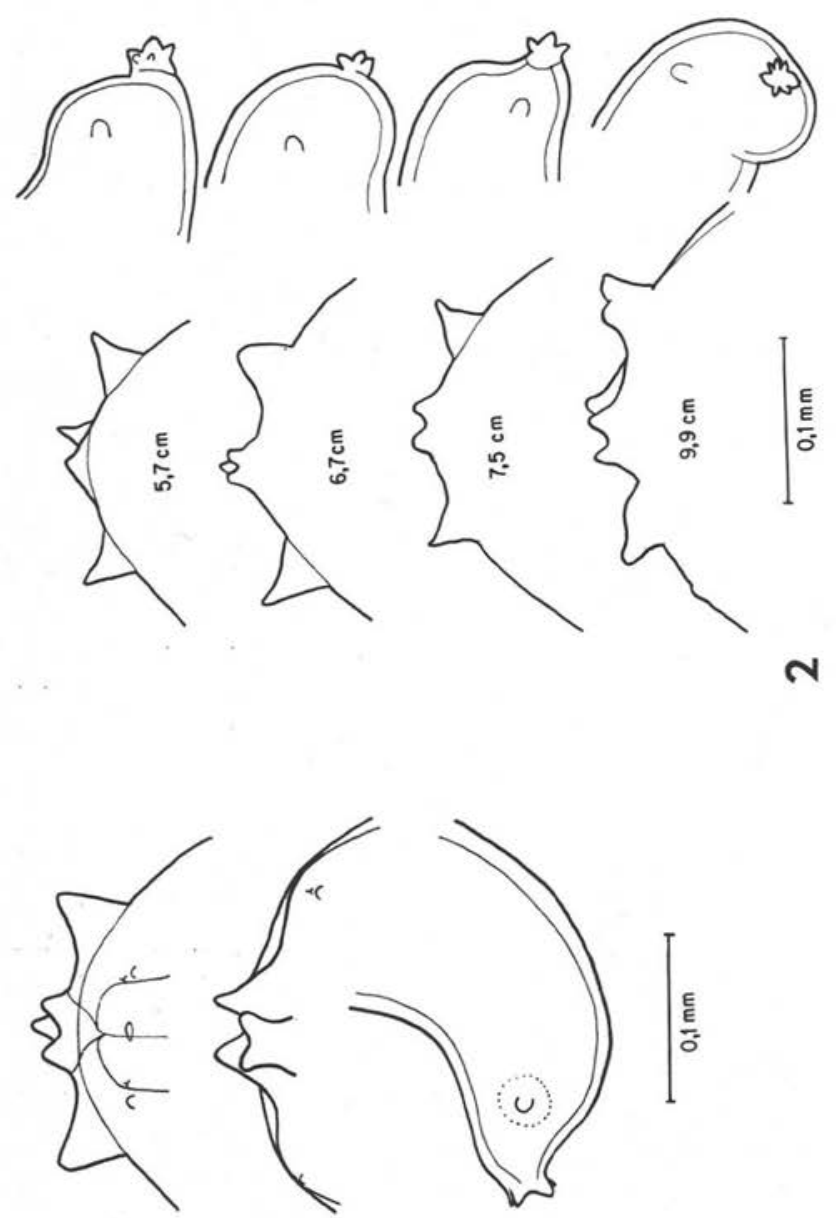

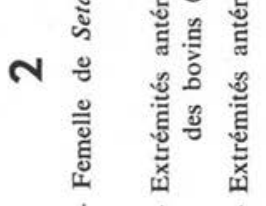

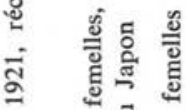

ㅇํㅇ ีㅜㅇ

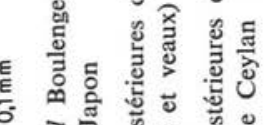

जี

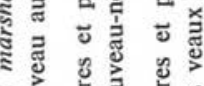

ปั

l 1

$\begin{array}{lll}\dot{1} & \dot{1} & \dot{1} \\ \dot{1} & \dot{4}\end{array}$
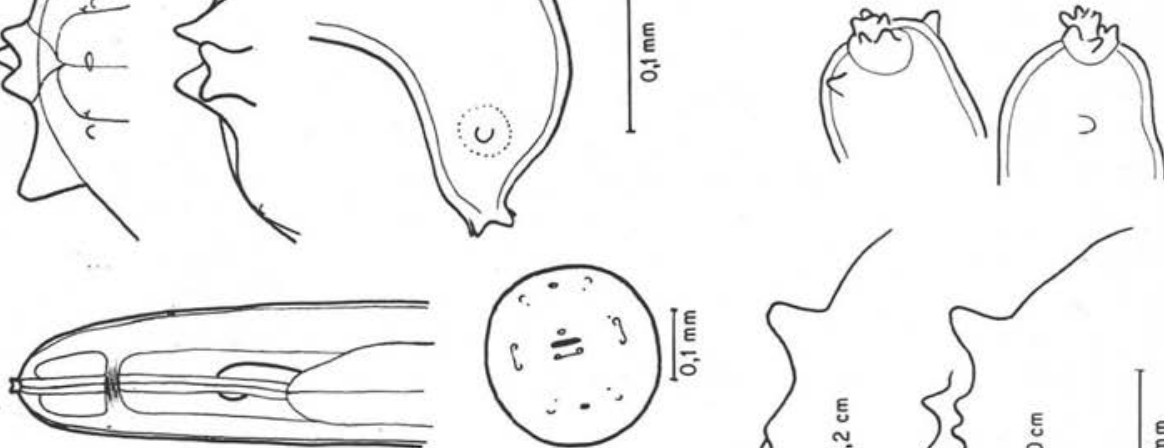

$\left(\begin{array}{c}\cdots \\ i \\ \cdots\end{array}\right]$
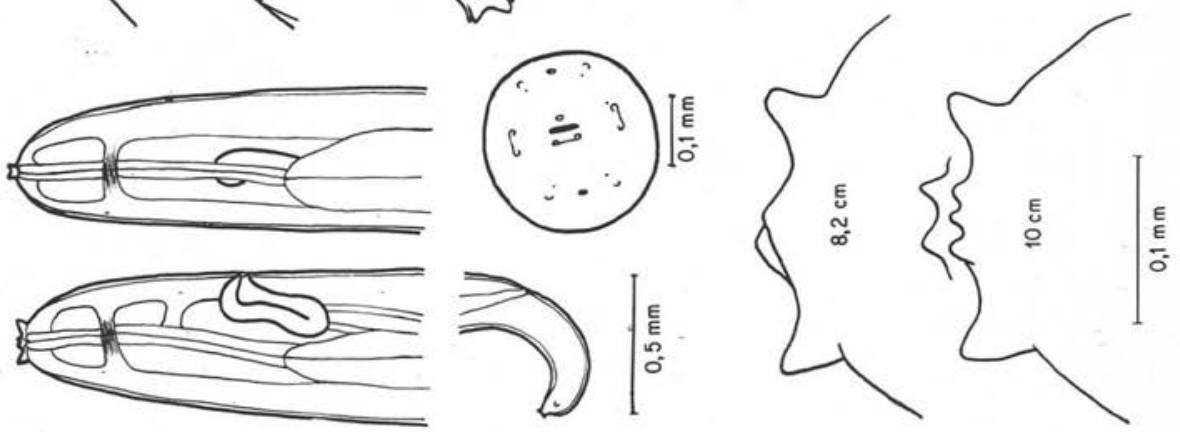

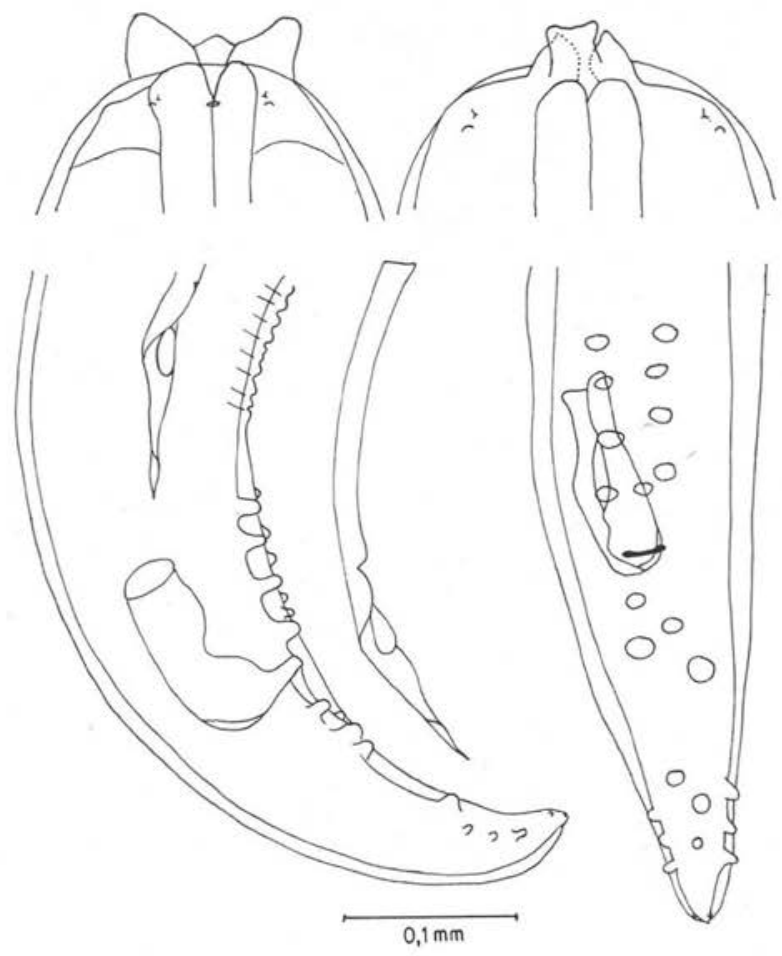

Fig. 4. - Mâle de Setaria marshalli, récolté au Japon

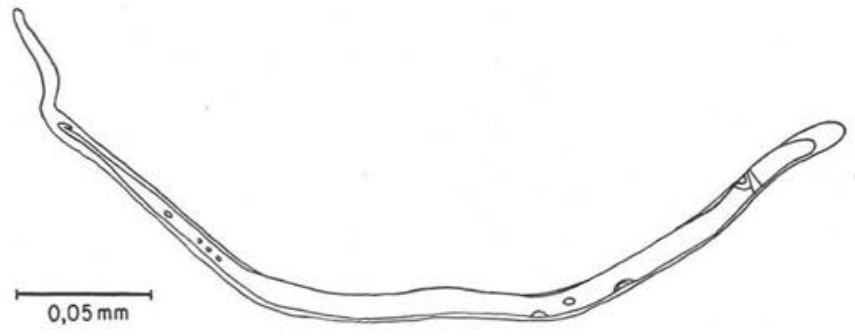

FIG. 5. - Microfilaire de Setaria marshalli 


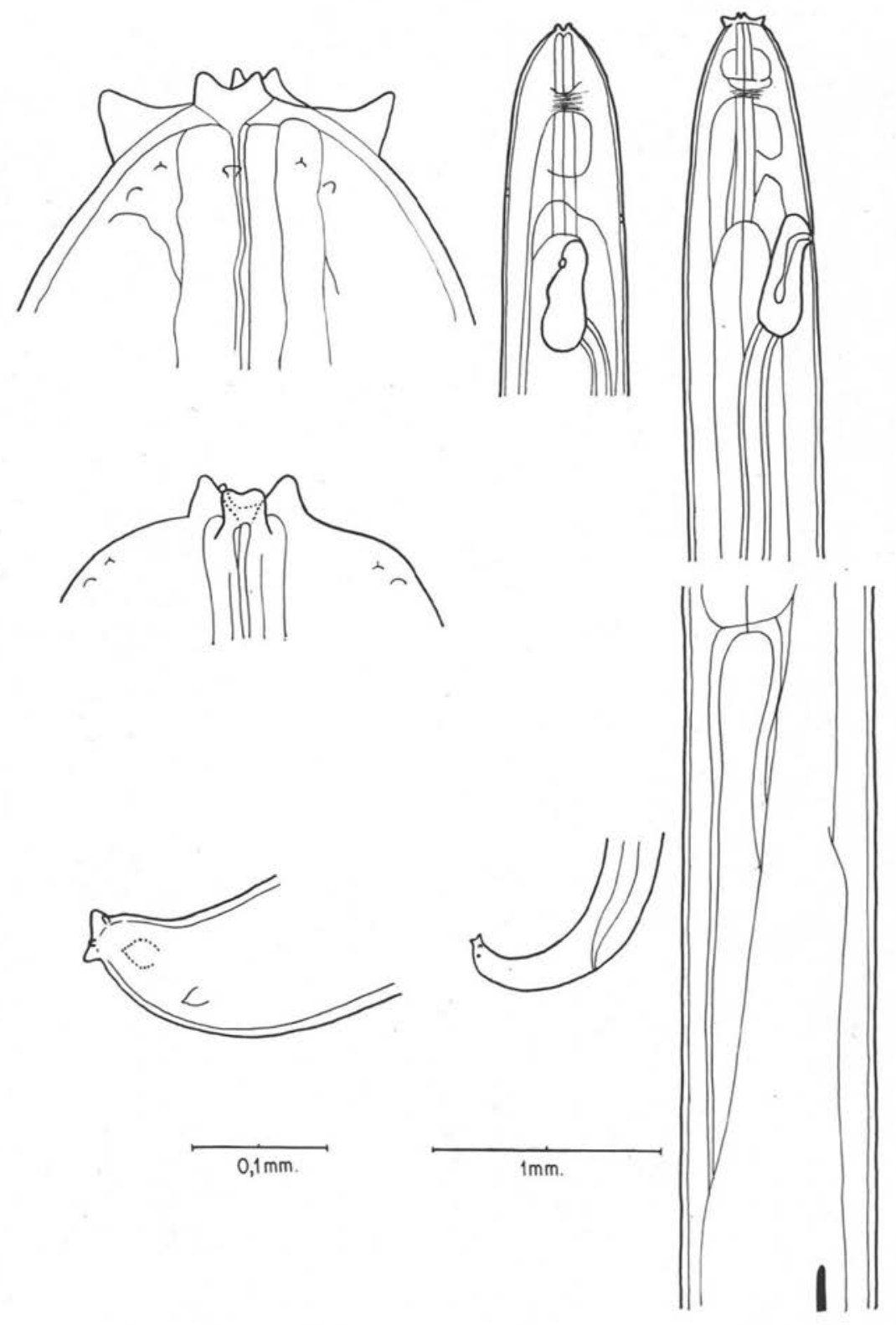

Fig. 6. - Femelle de Setaria marshalli pandei n. sous-esp. 

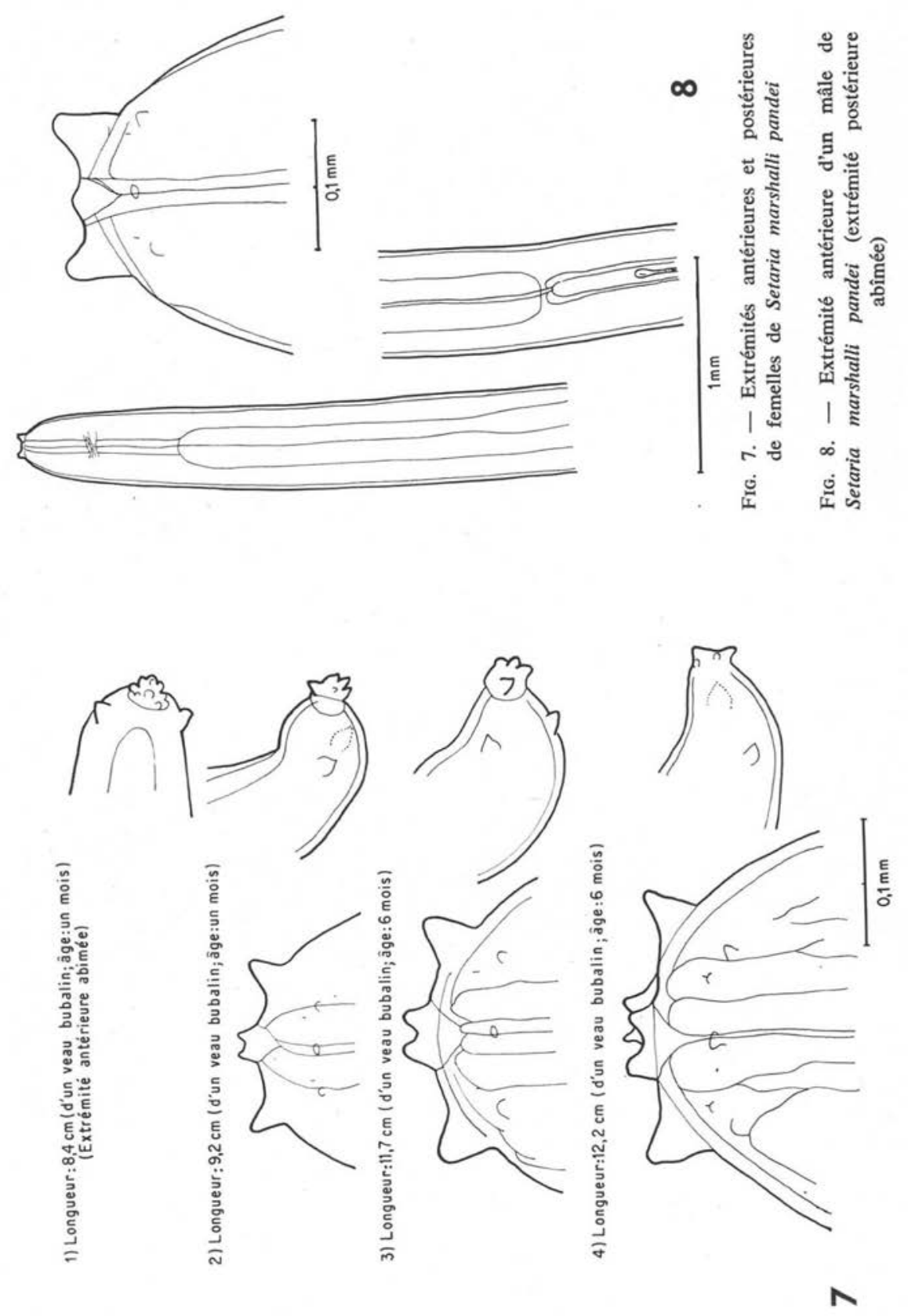

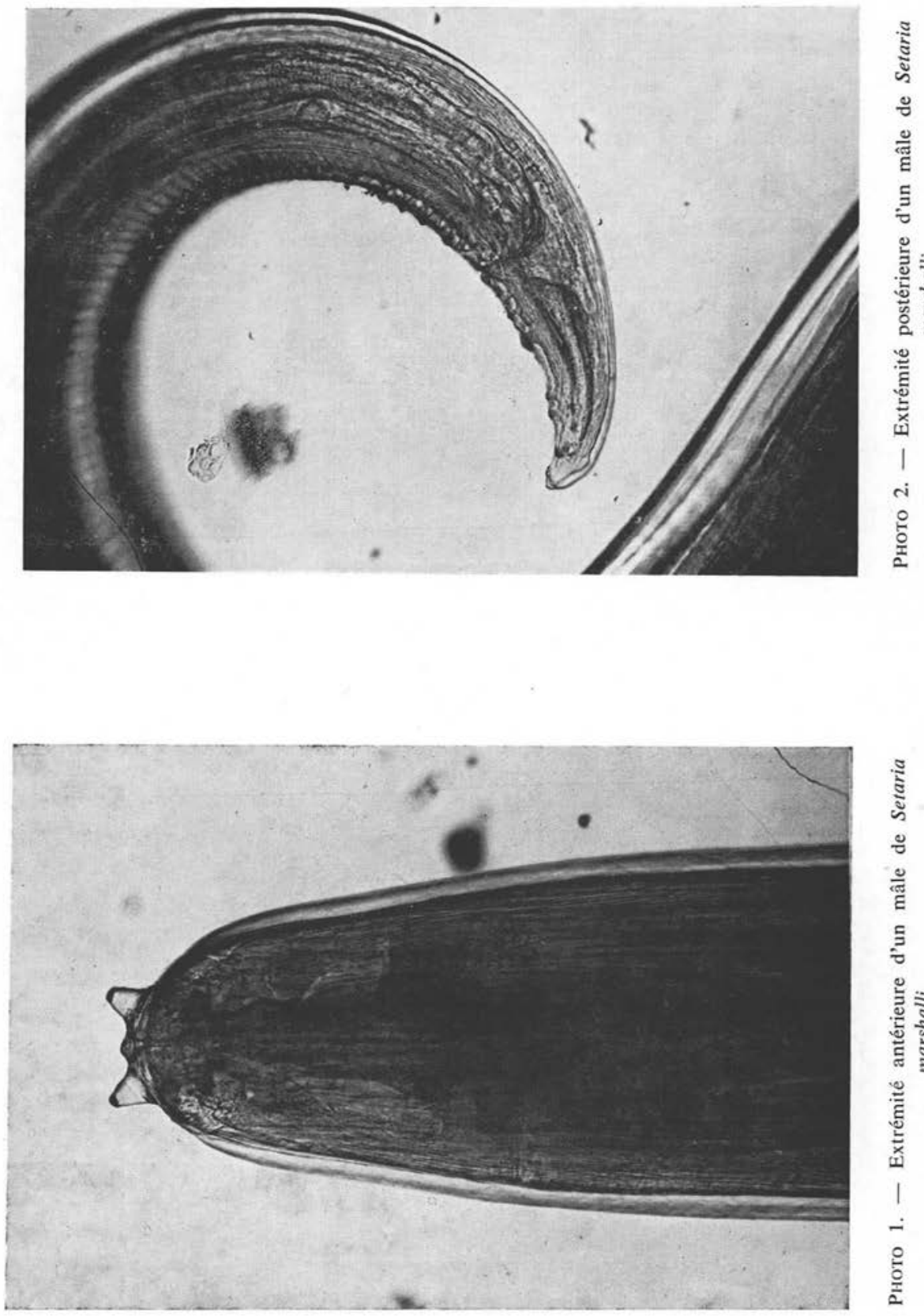


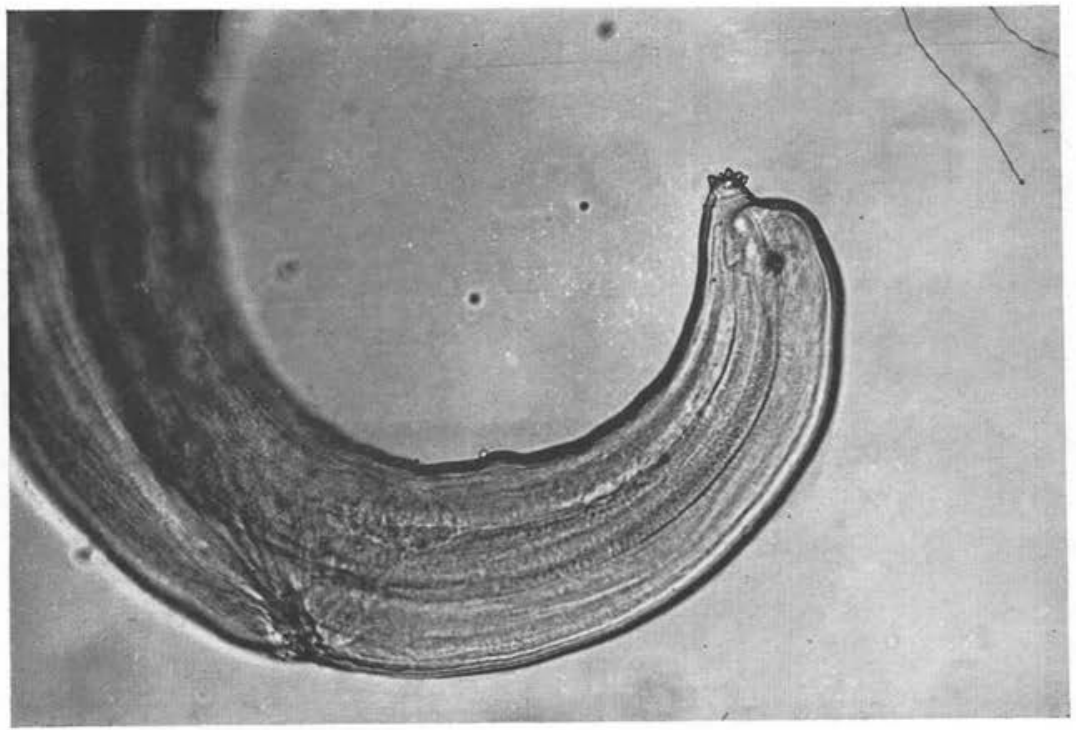

ะ

๕

ฐ

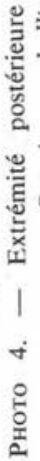

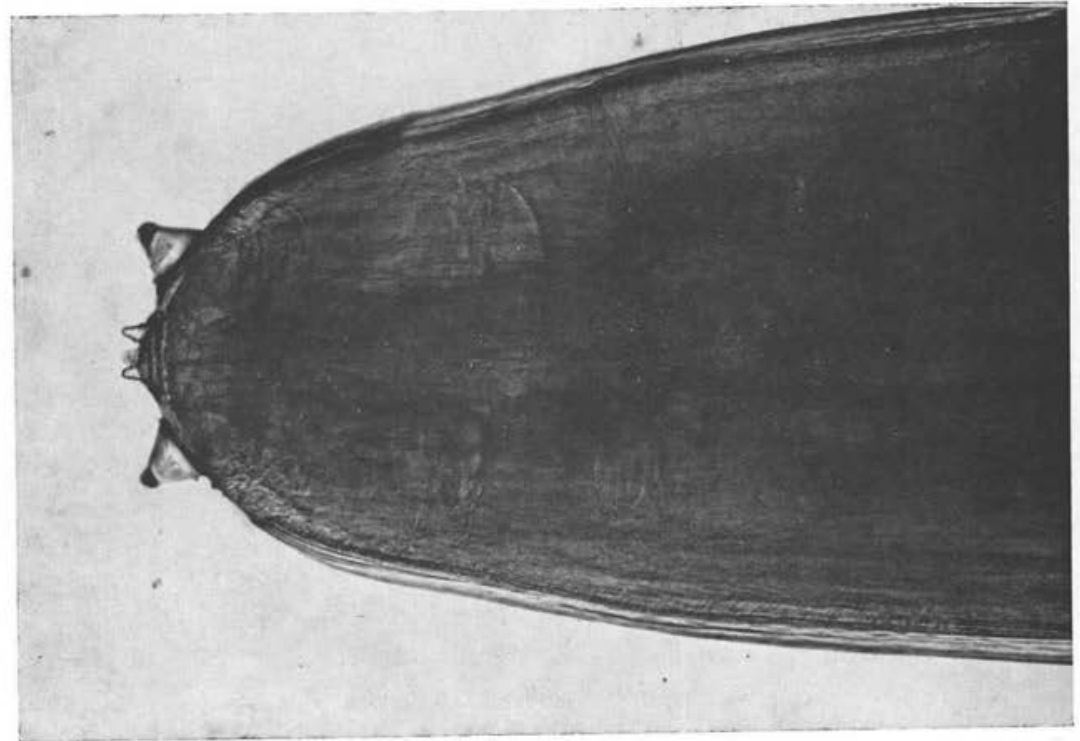



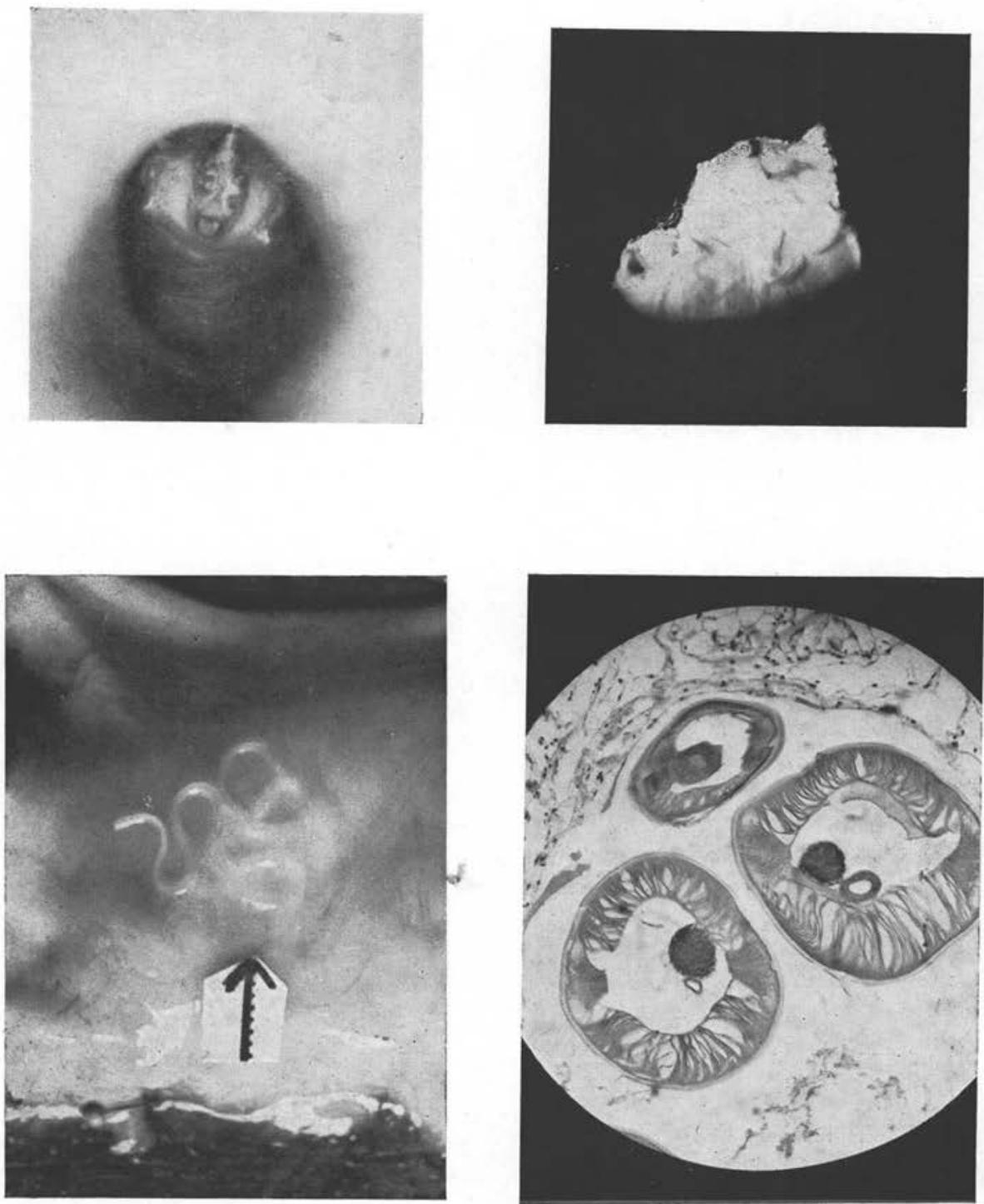

Pното 5. - Extrémité antérieure d'une femelle de Setaria marshalli (vue apicale)

Pното 6. - Un Setaria sous la séreuse du péritoine (récolté dans l'intestin grêle d'un jeune veau Рното 7. - Un autre Setaria sous la séreuse (d'un veau nouveau-né)

Рното 8. - Section histologique d'un Setaria dans la séreuse. Notez l'absence de réactions histologiques dans la sous-séreuse, autour d'un Setaria (veau nouveau-né) 

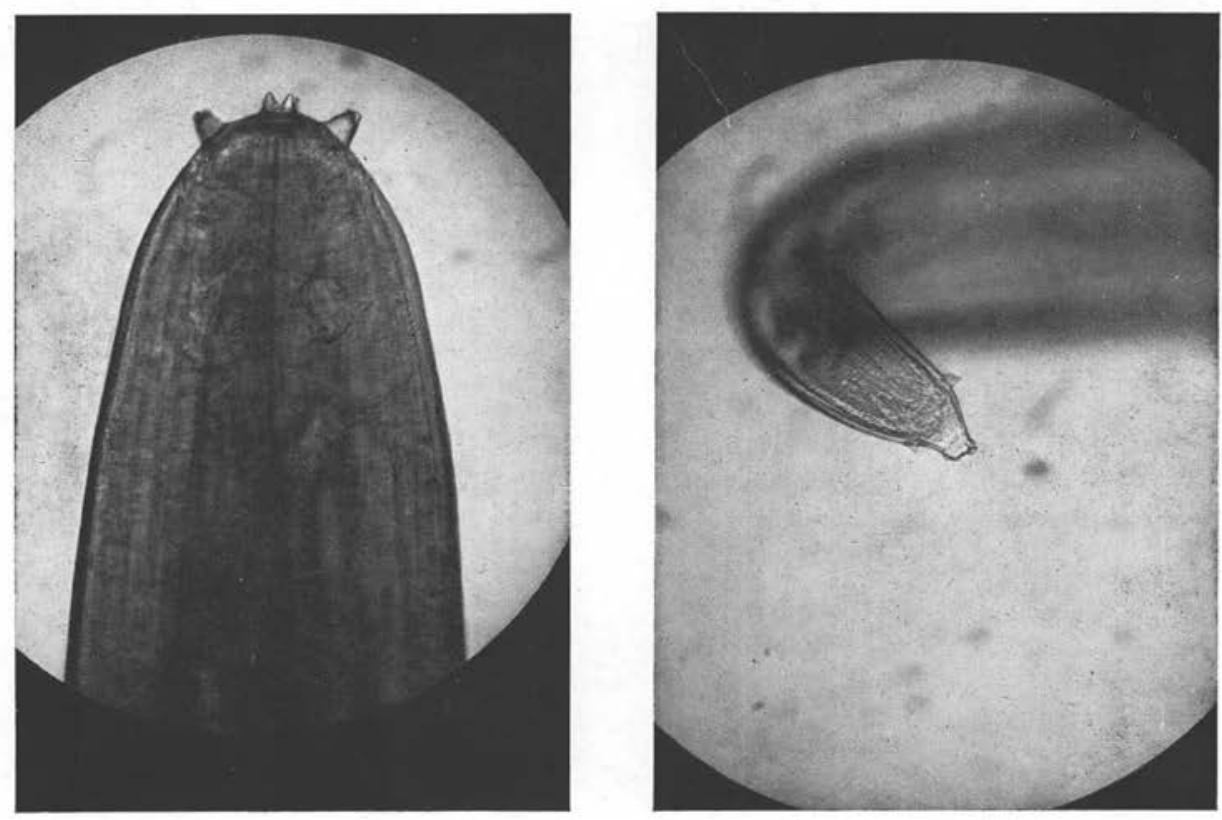

Рното 9. - Extrémité antérieure de Setaria marshalli pandei

Рното 10. - Extrémité postérieure de Setaria marshalli pandei

veaux nouveau-nés et chez les jeunes adultes, rarement chez les chèvres et les moutons, parfois chez les poulains.

La sous-espèce nouvelle signalée ici par nous ne pourra être décrite valablement que lorsque l'existence de Setaria marshalli sera admise sans contestation. C'est pourquoi nous nous efforcerons d'abord de justifier la validité de cette espèce, remettant à plus tard la discussion de la validité de sa sous-espèce.

\section{SETARIA MARSHALLI Boulenger 1921}

\section{Historique}

Cette espèce a été décrite pour la première fois par Boulenger en 1921, à partir d'une seule femelle trouvée dans un lot de Vers provenant de bœufs indigènes (WasseinBirmanie) et récoltés par le $\mathrm{D}^{\mathrm{r}}$ Marshall. Baylis considérait cette espèce comme un échantillon rétracté et abîmé de Setaria labiato-papillosa (Alessandrini, 1838, ou Perroneito, 1882), mais il n'était pas sûr de son diagnostic. Il semble que ce travail ait fortement influencé les travailleurs de langue anglaise. 
Oguni (1929) a signalé cette espèce dans sa liste de Vers provenant de bœufs indigènes, à Hiroshima, dans l'île principale du Japon (Honshu). En 1939-1943, l'étude des hôtes intermédiaires était effectuée par des savants japonais (surtout par le $\mathrm{D}^{r} \mathrm{D}$. Niimi), en Corée, parallèlement à une étude expérimentale sur le rôle de Setaria digitata, dans les «Paralysies des moutons et des chèvres ». La longueur des microfilaires avec leur gaine est de 360-380 $\mu$ pour $S$. marshalli et de 260-280 $\mu$ seulement pour $S$. digitata. Le moustique Anopheles hyrcanus sinensis est un hôte intermédiaire commun aux deux espèces, tandis que Armigeres obturbans ne transmet que la dernière espèce. Ces auteurs confirmaient aussi, par transmission expérimentale, que $S$. marshalli n'avait aucun rôle dans la paralysie lombaire des moutons et des chèvres, tandis que $S$. digitata la déterminait au contraire ; cette observation est en contradiction avec celle publiée plus tard en Russie par Cadenacii (1956). Au Japon, aucune observation n'est venue jusqu'à présent soutenir les assertions du travailleur russe.

Une observation intéressante était publiée par Asahi (1943), qui décrivait le mâle de $S$. marshalli pour la première fois ; cette espèce était trouvée chez des poulains sur une petite île de l'extrême sud du Japon. Chez un poulain âgé de cinq mois, 7 exemplaires ( 2 mâles et 5 femelles) étaient récoltés, tandis que chez un autre poulain âgé de onze mois, 4 spécimens (un mâle et trois femelles) étaient découverts mélangés avec $80 \mathrm{~S}$. equina et une femelle de $S$. digitata. Cette observation qui n'a pas encore été confirmée méritait, croyons-nous, d'être signalée ici.

Après la découverte de quelques Setaria chez les veaux nouveau-nés (Shoho et Nirasawa (1951), plusieurs travaux précisèrent que ces Setaria étaient surtout des $S$. marshalli et nous sommes maintenant tenté de penser que $S$. marshalli infecte normalement les animaux par la voie intra-utérine [voir Shoho (1955)].

Quelques cas d'infection par $S$. marshalli de la cavité péritonéale ou de la sousséreuse des moutons et des chèvres ont été rapportés, mais d'après nos observations personnelles au Japon et aussi à Ceylan, il devait s'agir surtout d'infestations anormales, en raison de l'absence constante chez ces hôtes de microfilaires dans l'utérus de la femelle alors stérile (œufs seulement) (pas d'observation faite chez les poulains).

Des mensurations précises ont été faites sur les microfilaires et les adultes par Hayashi et al. (1953), ainsi que par Taniguchi (1953).

L'observation effectuée en Russie sur la paralysie lombaire des moutons par $S$. marshalli (Cadenacii 1956) pose la question de savoir si $S$. marshalli en Russie n'est pas identique à l'espèce décrite en Asie.

\section{Critères diagnostiques}

Boulenger donne clairement les caractéristiques de l'espèce dans sa description d'une seule femelle: * The peribuccal ring elongated in dorso-ventral direction and very deeply notched laterally. The lip-like lateral projections exceptionally prominent and marked tooth-like, being themselves indented at their apices. The posterior extremity is rounded. The caudal appendage is bifurcated and situated very close to the posterior extremity of the body. » 
A notre avis, quelques précisions complémentaires à cette diagnose semblent nécessaires, ainsi : 1) pour « the lip-like lateral projections », ajoutons qu'elles présentent parfois une ou deux dents ou n'en présentent aucune (voir figures) ; - 2) pour \& the caudal appendage bifurcated and situated very close to the posterior extremity of the body », signalons qu'il est petit et rond, situé près de l'extrémité postérieure avec protubérance terminale bifurquée ou spinulée.

S. marshalli sera ainsi décrit de la manière suivante :

\section{Morphologie :}

Corps blanc, cylindrique, extrémité amincie légèrement enroulée (moins chez la femelle). Cuticule épaisse, recouvre le corps entier.

Bouche étirée ventro-dorsalement, étroite en vue apicale; elle est entourée d'un anneau chitinoïde ovalaire avec des projections proéminentes ventro-dorsales et latérales; les premières sont légèrement dentées tandis que les dernières sont surélevées selon le type Tholoïde ou Belonite pour les mâles, ou selon le type Belonite ou conique, avec une ou deux dents ou sans dent, en vue latérale, chez les femelles. Près de l'extrémité céphalique se trouvent 8 papilles et 2 amphides; 4 papilles latéro-médianes arrondies et distinctes, et 4 papilles médio-médianes, situées un peu antérieurement, plus petites et saillantes. Les amphides très petites sont situées latéralement. Diérides symétriques situées un peu en arrière de l'anneau nerveux.

Esophage antérieur musculeux, court, suivi par le postérieur, glandulaire, plus long et plus large, communiquant avec l'intestin, moins large, mais à la lumière agrandie au début.

Femelle (matériel type provenant d'un veau au Japon) : $92 \mathrm{~mm}$ de long, largeur maximum : 0,693 $\mathrm{mm}$. L'une des projections latérales de l'anneau chitinoïde péribuccal est dentée, l'autre non. Apex des projections dorsales et ventrales : 0,16 mm chacune, anneau nerveux situé à $0,306 \mathrm{~mm}$, de l'extrémité antérieure, diérides, un peu asymétriques, à $0,58 \mathrm{~mm}$ et à $0,71 \mathrm{~mm}$, respectivement de l'apex.

Esophage 8,5 mm; portion antérieure: $1,0 \mathrm{~mm}$, partie postérieure: 7,5 $\mathrm{mm}$. Vulve à $0,839 \mathrm{~mm}$ de l'apex. L'appareil génital commence avec la portion du sphincter piriforme, de l'ovéjecteur à l'intérieur duquel la lumière génitale est spiralée. Il se poursuit par un tube plus large postérieurement, de $4 \mathrm{~mm}$ de long, qui s'élargit graduellement et se transforme en utérus, rempli d'innombrables microfilaires au niveau de la bifurcation, à $8,55 \mathrm{~mm}$ de l'apex; la bifurcation se trouve approximativement à l'extrémité de l'œsophage chez tous les exemplaires, soit un peu en avant, soit un peu en arrière. Opisthodelphie. La portion terminale de l'utérus se mêle aux oviductes et aux ovaires à la partie postérieure du corps. L'anus est à $0,58 \mathrm{~mm}$ de l'extrémité postérieure. Une paire d'appendices latéraux, petits et ronds, se trouvent à $0,06 \mathrm{~mm}$ de l'apex de la queue qui se courbe légèrement ventralement et se termine en une protubérance distale munie d'épines relativement distinctes (parfois certaines épines apparaissent comme bifurquées).

Les microfilaires avec leur gaine, prises dans l'utérus, sont longues de 0,36 à $0,42 \mathrm{~mm}$ environ. 
MALE (matériel prélevé sur un jeune bovin au Japon) : $55 \mathrm{~mm}$ de long, largeur maximum : $0,5 \mathrm{~mm}$. Un anneau chitinoïde péribuccal distinct ; projections latérales de type Theloïde en vue latérale et non dentées chez tous les exemplaires; apex des projections ventrale et dorsale : $0,092 \mathrm{~mm}$. Anneau nerveux situé à $0,306 \mathrm{~mm}$ et diérides à $0,661 \mathrm{~mm}$ et à $0,726 \mathrm{~mm}$ respectivement de l'extrémité antérieure. Les papilles et les amphides disposées comme chez les femelles. Esophage : 7,564 mm de long; partie antérieure : $0,039 \mathrm{~mm}$; partie postérieure : 0,725 mm. Spicules inégaux : le plus long, $0,303 \mathrm{~mm}$; le second, court, plus épais, avec un orifice étroit dirigé vers le cloaque. Extrémité caudale $0,148 \mathrm{~mm}$ de long se terminant en pointe mousse, légèrement bifurquée ou non. Respectivement 4 paires de papilles pré- ou post-cloacales. La première et la troisième paire de papilles post-cloacales sont peut-être un peu asymétriques et la quatrième, plus petite, est située près de l'extrémité postérieure. Postérieurement, au niveau de la $3^{\circ}$ paire de papilles post-cloacales, il y a 3 paires d'appendices latéraux petits, mais à peu près de la même largeur.

Hôte: Bovins (nouveau-nés et veaux) (hôte type: Bos indicus). Les chèvres et les moutons sont des hôtes anormaux.

HôTE INTERMÉdiaIRE: Anopheles hyrcanus sinensis en Corée et au Japon.

Localisation: cavité péritonéale pour le ver adulte; tissu connectif et sous-séreuse de la cavité péritonéale pour le jeune ver).

MatéRIel et Lieu D'origine: 9 femelles récoltées chez des veaux âgés de moins d'un mois disséqués en automne au Japon ; 3 femelles provenant de veaux sacrifiés en hiver et au printemps au Japon; 2 mâles et 1 femelle récoltés chez un jeune bovin japonais; 1 femelle provenant de Bos indicus à Ceylan (récolté par le $\mathrm{D}^{\mathrm{r}}$ C.-P. Pillai); 1 mâle et 4 femelles provenant de chèvres de Jamna Pari, au Nord de Ceylan.

\section{Discussion}

Avec les appendices latéraux «petits et ronds » observés par nous et compte tenu du «bifurcated caudal appendice» et de l' «indented lateral peribuccal chitinous ring » signalés par Boulenger, ces critères morphologiques des femelles semblent suffisants pour différencier $S$. marshalli de toutes les autres espèces du genre Setaria Viborg 1793.

En ce qui concerne les appendices latéraux petits et ronds, ils peuvent facilement échapper à l'attention lorsque l'on travaille sur un exemplaire unique ou mal fixé. Cette forme des appendices latéraux est particulière, car ainsi que nous le verrons ci-dessous, c'est un caractère différentiel entre cette espèce et la sous-espèce nouvelle observée par nous. La même différence existe nettement entre Setaria de Rangifer tarandus (S. tundra Rajewsky et Issaitschkow, 1928) dans l'île de Mundjuk en Océan arctique et Setaria d'Odocoileus virginianus encore incomplètement étudié en Amérique du Nord (la première a des appendices ronds, tandis que la seconde possède des 
appendices pointus, caractère non signalé parmi les autres caractères différentiels dans un travail récent [Yeh 1959]).

La diversité des indentations des projections latérales de l'anneau chitinoïde péribuccal des femelles et l'absence de denticulations homologues chez les mâles sont visibles sur les figures incluses dans ce texte.

La longueur des microfilaires de cette espèce est sensiblement plus grande que celle des microfilaires de $S$. digitata et de $S$. labiato-papillosa, deux espèces communément observées chez les bœufs domestiques, la première surtout en Asie, aux Indes, la seconde dans toutes les autres parties du monde. C'est un critère morphologique utile pour identifier cette espèce.

La description que nous donnons ci-dessus pour le mâle est presque identique à la description originale de Asahi (1943). Certains points, tels que : la non-denticulation des projections latérales de l'anneau chitinoïde péribuccal et l'identité relative de largeur des 3 paires d'appendices latéraux, sont importants pour différencier cette espèce des autres espèces du genre. On sait à présent que les appendices latéraux ne sont semblables que chez $S$. tundra et $S$. capreola (Cadenacii et Garcavi 1957), Shoho (1959).

En ce qui concerne le mode de pénétration chez les nouveau-nés, nous pensons que la voie normale pour cette espèce est identique à celle que nous avons observée pour Toxocara canis (Shoho 1955) (1). Déjà quelques observations sur des infections intrautérines par un Setaria ont été publiées; par exemple, pour S. labiato-papillosa (Alessandrini, 1838 ou Perroncito, 1882) en Russie (Cadenacii, 1938), S. cervi aux Philippines (Rofuerzo 1952), et pour un Setaria non identifié chez un fœtus abortif de cheval au Japon (Nirasawa 1938). S. marshalli nous fournit, pour la première fois chez les Nématodes, une illustration de ce type d'infestation. La première partie du cycle de développement se fait chez un Insecte, hôte intermédiaire, et l'infestation de l'hôte vertébré normal s'effectue par la voie intra-utérine.

Bien que cette opinion soit souvent considérée comme absurde, le phénomène d'infection prénatale par un Nématode est connu depuis longtemps : au siècle dernier, une observation de ce genre sur des microfilaires chez un fœtus de Mustelus laevis a été publiée par Leidig (1851). Pour Davaine (1877), l'infection intra-utérine n'est pas certaine chez l'homme, mais possible quoique rare chez les animaux. Leuckart (1879-1886) s'est rallié à l'opinion de Davaine ; il avait en effet trouvé des Nématodes d'environ $0,5 \mathrm{~mm}$ de long dans le sac épicardique, la cavité crânio-vertébrale et le liquide amniotique de foetus provenant d'une femelle gestante de Lacerta agilis chez laquelle il n'a pu découvrir de vers adultes. Il en déduisit que l'infection s'était propagée par la voie intra-utérine; mais ce cas ne semble pas avoir été analysé sérieusement. Toutefois, c'est peut-être en raison de ce mode occulte de pénétration que l'attention des travailleurs n'a pas été attirée sur ce Ver. Une nouvelle expérimentation nous permettra de conclure sur ce point.

(2) Cette opinion est discutée dans le travail de Sprent (1955). Nous croyons néanmoins que le mode de pénétration de $T$. canis chez son hôte normal au Japon est pratiquement la voie intrautérine. 
Dimensions de Setaria marshalli

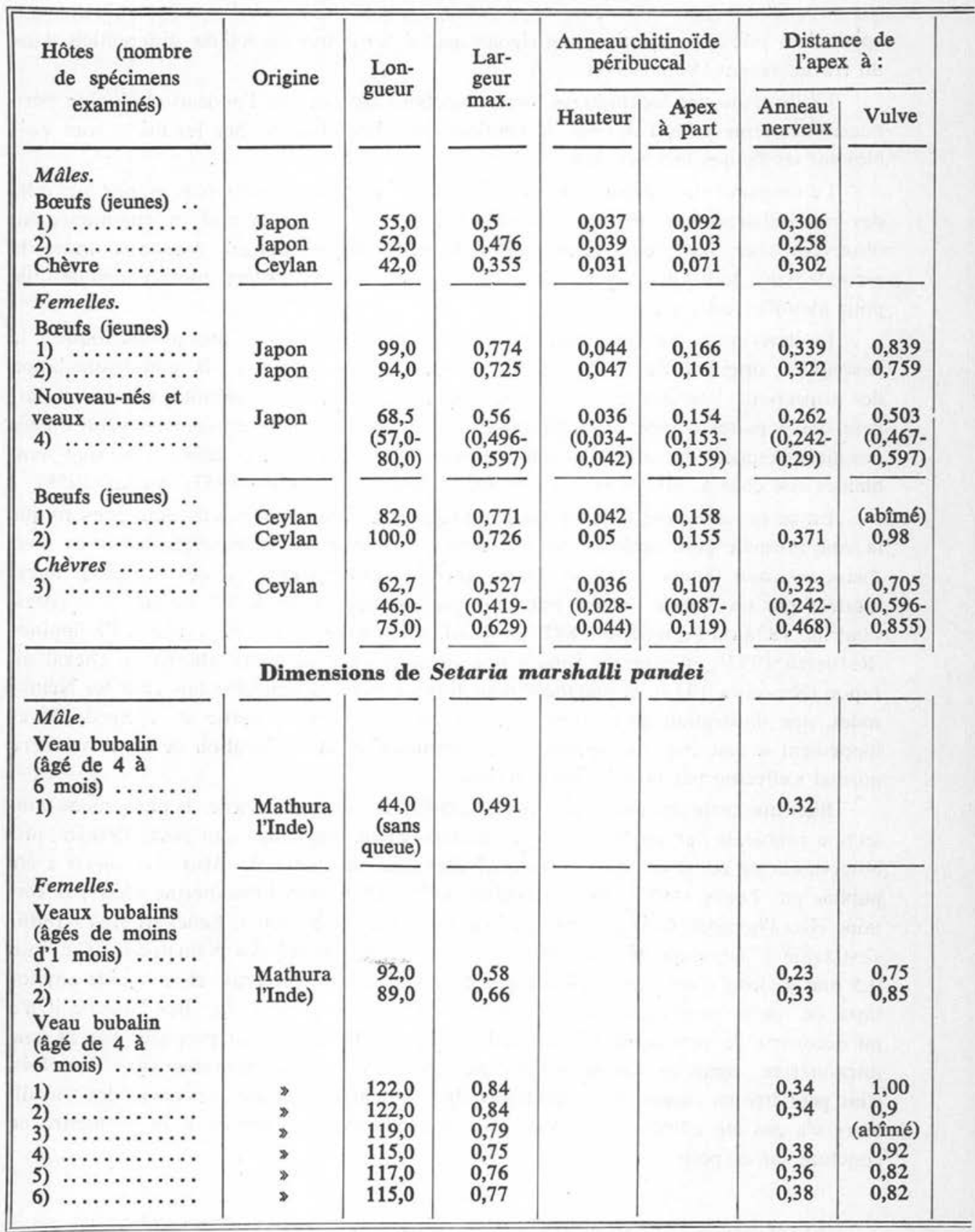

Les chiffres moyens, pour les exemplaires provenant de veaux nouveau-nés au Japon et de chèvres à Ceylan, sont suivis par les dimensions minima et maxima observées. 


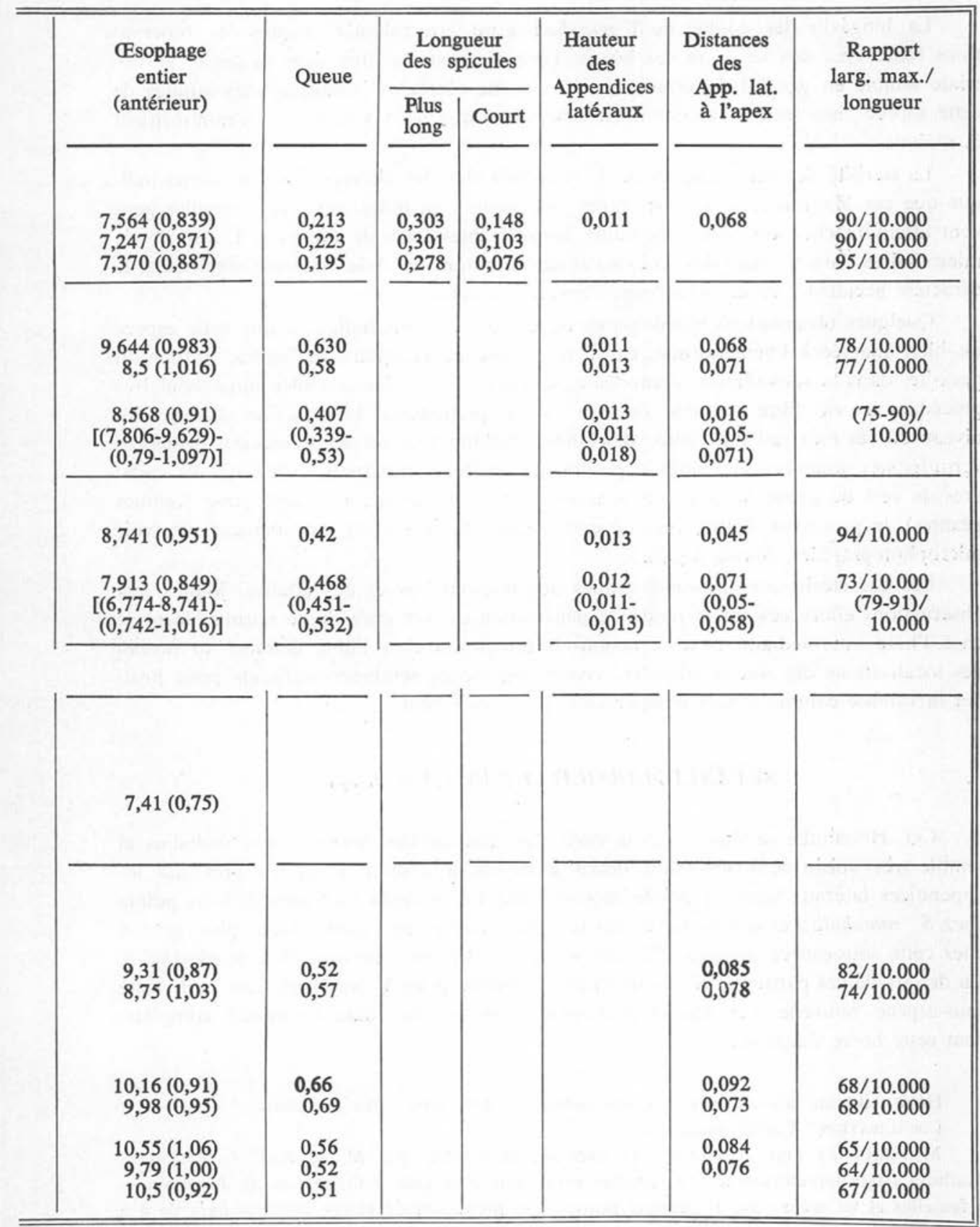

Certains chiffres manquent, la mauvaise fixation n'ayant pas permis des mensurations correctes. 
La longévité des adultes de $S$. marshalli a pu être calculée d'après des observations faites chez des veaux et des bœufs. Leur vie sexuelle libre dans la cavité péritonéale semble en général d'une année, car nous ne récoltons jamais de vers adultes de cette espèce chez les bœufs adultes en Asie, alors que nous y trouvons communément S. digitata.

La stérilité des vers femelles de $S$. marshalli chez les chèvres et les moutons indique que ces Mammifères sont des hôtes anormaux; en outre, ces Vers femelles peuvent atteindre chez ces hôtes des tailles considérables (plus de $60 \mathrm{~mm}$ ). L'absence de microfilaires dans le sang des chèvres et des moutons en Asie confirme également le caractère accidentel de ce parasitisme chez ces animaux.

Quelques observations histologiques effectuées par nous indiquent que cette espèce est bien adaptée à l'hôte normal, car il n'y a aucune réaction histologique autour du parasite, dans la sous-séreuse péritonéale, au cours des stades juvéniles intra-tissulaires précédant la vie libre sexuelle dans la cavité péritonéale. La réaction du tissu au niveau du ver mort est différente; des études histologiques sur les substances brunâtres vermiformes attachées à la séreuse péritonéale du bœuf montrent qu'il s'agit de cadavres de vers du genre Setaria avec réaction lympho-réticulaire et phagocytose (cellules géantes), le processus d'attaque et de destruction des vers étant parfaitement net (voir microphotographies, Shoho 1955).

Les caractéristiques morphologiques des microfilaires et des adultes, jointes aux observations effectuées sur le mode de pénétration du ver chez l'hôte normal, le cycle chez l'hôte intermédiaire et les réactions histologiques chez l'hôte définitif au niveau des localisations des stades juvéniles, vivants ou morts, semblent suffisants pour justifier la validité comme espèce indépendante de $S$. marshalli.

\section{SETARIA MARSHALLI PANDEI n. s. sp.}

Cet Helminthe se situe dans la cavité péritonéale des jeunes veaux bubalins et semble très voisin de $S$. marshalli, décrit ci-dessus, à la seule exception près que les appendices latéraux sont en pointe mousse chez les femelles (arrondis et sans pointe chez $S$. marshalli), et que la taille des femelles adultes est sensiblement plus grande chez cette sous-espèce (jusqu'à $122 \mathrm{~mm}$ au lieu de $100 \mathrm{~mm}$ environ chez $S$. marshalli). En dehors de ces particularités, la description donnée pour $S$. marshalli vaut pour cette sous-espèce nouvelle. Les figures et mensurations fournies dans ce travail complèteront cette brève diagnose.

Hôte : Bovins nouveau-nés et veaux bubalins (Hôte type: Bos bubalus).

LOCALISATION: Cavité péritonéale.

Matériel et Lieu D'origine: 11 exemplaires récoltés par M. le Prof. G.-P. Pande, Mathura (Inde septentrionale); 4 femelles provenant d'un veau bubalin âgé de 4 semaines ; 6 femelles et un mâle (sans l'extrémité postérieure) provenant de veaux bubalins âgés de 4 à 6 mois environ. 
Note. - Un lot de Setaria provenant de veaux bubalins âgés de 4 à 6 mois comportait un autre spécimen de femelle de Setaria que nous avons identifié à $S$. bubali (Rudolphi 1819); longueur $89 \mathrm{~mm}$. Dans ce cas, nous pensons que l'animal a dû s'infester après sa naissance. L'étude de cette dernière espèce de Setaria suivra dans un avenir prochain.

\section{Conclusion}

Setaria marshalli Boulenger 1921 est présent chez les veaux en Asie.

Les Setaria trouvés chez des veaux bubalins en Inde septentrionale appartiennent à une sous-espèce nouvelle, désignée par nous sous le nom de $S$. marshalli pandei.

\section{Références}

AsıHI O., 1943. - Sur des filaires de la cavité péritonéale des chevaux. Kachikueisei Kyokaiho. Rapport Soc. Hyg. Ani., 11, 61. (Japonais).

BAYLIS H. A., 1936. - On the nomenclature and synonymy of the Nematode Setaria labiatopapillosa. Ann. Trop. Med. Parasit., 30, 293.

Boulenger C. L., 1921. - On some filarid parasites of cattle and other ruminants. Parasit., $12,341$.

Bush D. L., 1951. - Lumbar paralysis of ovine species in Japan, reportedly by Setaria digitata. J. Am. Vet. Med. Ass., 118, 388.

Davaine C., 1877. - Traité des Entozoaires et des maladies vermineuses de l'homme et des animaux domestiques. Paris : J.-B. Baillière et fils.

Fülleborn F., 1920. - Ueber die Anpassung der Nematoden an den Parasistismus und den Infektionsweg bei Askaris und anderen Fadenwürmern des Menschen. Arch. Schiff. Trop. Hyg., 24, 340.

Japanese Research Commission in Korea, 1939-1944. - Transmission Experiment of Setaria marshalli in sheep. 4th Report (1943). (Japonais).

KADENACIr A.-N., 1938. - (Extrait en Jahresb. Vet. Med., 66, 58, 1940).

—, 1956. - Setaria dans les moutons; Biologie du parasite causal, C.R. Acad. Sci., U.R.S.S., 107, 191 (Russe).

— et Garkavi B. L., 1957. - Setaria capreola. Trudy Omsk. Vet. Inst., 15, 237 (Russe).

Leuckart R., 1879-1886. - Die Parasiten des Menschen und die von ihnen herrührenden Krankheiten. Leipzig und Heidelberg ; C. W. Winterische Verlag.

Oguni H., 1929. - Recherche sur la maladie dermique «Wahi » et « Kosé » des bœufs. Chuoiji., 42, 633. (Japonais).

Sato K. et Hayashi S., 1953. - Etude sur le genre Setaria, parasitaire au bœuf. Jap. J. Vet. Med., 116, 761. (Japonais).

Sноно C., 1955. - Helminthic diseases of the central nervous system and intra-utérine foetal infection by Helminths. Rev. Ibêr. Parasit., Tomo Extra., 927. 
-, $1958 a$. - On the identity of Setaria spp. from the abdominal cavity of Ceylon spotted deer, Axis A. ceylonensis Fitzinger. Ceylon Vet. J., March-June, 1.

-, 1958 b. - Studies on the identity of Setaria species from the peritoneal cavity of American moose (Alces A. gigas) and white tailed deer (Odocoileus virginianus). Ceylon Vet. J., Sept.-Déc., 43.

-, 1959. - Die Setarien vom schweizerischen Reh, Capreolus c. Rev. Suisse de Zool., 1, 233.

- et Nirasawa T., 1951. - Données inédites. (Voir Shoho, 1955).

SPRENT J. F. A., 1955. - On invasion of the central nervous system by Nematodes. I. Incidence of pathological significance of Nematodes in the central nervous system. Parasit., 45, 41.

-, 1958. - Observation on the development of Toxocara canis (Werner, 1782) in the dog. Parasit., 48, 184.

Thwarte J. W., 1927. - The genus Setaria. Ann. Trop. Med. et Parasit., 21, 427.

TANiguchi M., 1953. - Sur Setaria. Jap. J. Vet. Med., 116, 775. (Japonais).

Yamagrwa S. et Shoнo C., 1944. - Zur Pathologie der Setariosis der Schafe (eine neurohistologische Studie). Jap. J. Vet. Sci., 6, 413.

YeH L. S., 1959. - A revision of the Nematode Genus Setaria Viborg, 1795, its host-parasite relationship, speciation and evolution. J. Helm., 33, 1. 